\title{
Sprachwissenschaft
}

\author{
Anna Bajerowska \\ ORCID: 0000-0002-2894-8165
}

Universität Warszawa, Warszawa

DOI: $10.19195 / 0435-5865.144 .10$

\section{Emergenz, Spezifizität und Entropie im Lichte der anthropozentrischen Linguistik}

\begin{abstract}
s
Der vorliegende Artikel thematisiert das Problem der (Fach-)Wissensgenerierung vor dem Hintergrund der anthropozentrisch aufgefassten Denotationsproblematik. Der erste Teil widmet sich dem auf neurobiologischen Erkenntnissen basierenden und anthropozentrisch begründeten Modellvorschlag sprachgebundener (Fach-)Wissensgenerierung. Im Mittelpunkt der Erwägungen steht die konzeptuelle Verbindung von Emergenz und anthropozentrischen Annahmen im Kontext linguistischer Fragen. Im Fokus des zweiten und dritten Teils steht die Übertragung des Spezifizität- und Entropie-Begriffs auf das Gebiet der anthropozentrischen Linguistik. Es ist hervorzuheben, dass die hier präsentierten Überlegungen einen vorläufigen Charakter haben.
\end{abstract}

Schlüsselwörter: anthropozentrische Linguistik, Emergenz, Spezifizität, Entropie, Textdenotation

\section{Emergence, specificity and entropy in the light of anthropocentric linguistics}

The aim of this paper is twofold. First, a concept comprising a preliminary, emergence-based model of generating (specialized) knowledge will be outlined, which was built across anthropocentric linguistics, selected concepts of emergence and research in the field of neurobiology and which is aimed at offering a possible solution to the problem of the interactions between language and knowledge generating. Second, a conceptual reformulation of the terms specificity and entropy in the light of the anthropocentric theory of human languages will be proposed. It has to be highlighted that the present considerations are preliminary and require a more solid neurobiological foundation.

Keywords: anthropocentric linguistics, emergence, specificity, entropy, denotation

Anna Bajerowska, Uniwersytet Warszawski, Wydział Lingwistyki Stosowanej, Szturmowa 4, p. 510, 02-678 Warszawa, Polen, E-Mail: a.b.bajerowska@uw.edu.pl

Reveived: 7.10.2018, accepted: 9.04.2019

Germanica Wratislaviensia 144, 2019

(C) for this edition by CNS 


\section{Sprachbedingte (Fach-)Wissensgenerierung im Spiegel der Emergenz}

Den Ausgangspunkt für die hier präsentierten Erwägungen bildet die Behauptung von Sambor Grucza $(2008,2013)$, die besagt, dass der Existenz mancher Fachwissensbestandteile konkreter Menschen ihre Fachsprachenkenntnisse zugrunde liegen (S. Grucza 2013: 31, Vgl. S. Grucza 2008):

Die Rolle der Fachsprachen erschöpft sich nicht nur in der kommunikativen Funktion innerhalb verschiedener Fachgemeinschaften. Fachsprachen sind nicht ausschließlich kommunikative Werkzeuge ,ihrer“ Fachgemeinschaften. Sie erfüllen nicht nur eine kommunikative Funktion, indem durch sie Fachtexte produziert und rezipiert werden können, wodurch auf der Seite des Textproduzenten Fachwissen ausgedrückt und auf der Seite des Textrezipienten dieses (re) konstituiert werden kann. Fachsprachen sind in erster Linie kognitive Werkzeuge der jeweiligen Fachgemeinschaften, da sie ihren Mitgliedern als Werkzeuge der Facherkenntnis dienen. Mit anderen Worten: Fachsprachen erfüllen eine kognitive Funktion, da dank ihnen neues Fachwissen gebildet und altes umorganisiert werden kann.

Dem anthropozentrischen Ansatz zufolge stellt das spezifische Zusammenspiel zwischen den im Gehirn eines Individuums internalisierten sprachlichen Eigenschaften und Wissensgenerierungsprozessen eine konstitutive Funktion der Fachsprachen dar. Demnach nehme ich an, dass Fachwissen aus Fachsprachenkenntnissen emergieren kann ${ }^{1}$. Eine unerlässliche Voraussetzung für eine erfolgreiche Übertragung bestimmter sprachbezogener Ansätze auf das Gebiet des Emergentismus stellt eine theoretisch untermauerte Diagnose relevanter ontologischer Implikationen dar. Im Hinblick auf die Ontologie sprachlicher Eigenschaften der Menschen sowie auf die Textontologie, basieren die folgenden Erwägungen auf den Grundannahmen der anthropozentrischen (Fach-)Sprach(en)- und Texttheorie, aufgestellt entsprechend von Franciszek Grucza $(1983,1993,1997)$ und Sambor Grucza $(2004,2008,2013)$. Eine besondere Relevanz besitzt in dieser Hinsicht die auf dem anthropozentrischen Grund vorgenommene, klare Unterscheidung zwischen der denotativen Textebene und der Ebene von externalisierten Äußerungen d.h. der physikalischen (substanziellen) Textebene. Vor diesem theoretischen Hintergrund werden Textdenotate als mentale Größen angesehen, die infolge sprachgebundener Hirngewebe-Aktivität in den Gehirnen konkreter Sprecher-Hörer erzeugt werden. Den externalisierten Äußerungen gegenüber sind sie als autonome Entitäten zu betrachten:

Hinzugefügt sei noch, dass externalisierte Äußerungen als relativ autonome physikalische Objekte angesehen werden können. Unter funktionalem Gesichtspunkt besitzen sie allerdings keine sprachliche Selbständigkeit. Die inhärenten Komponenten von externalisierten Äußerungen stellen nur diejenigen physikalischen Eigenschaften dar, die ihre substantielle Identifizierung und Differenzierung möglich machen (es handelt sich um die Möglichkeit, ihre phonemische, graphemische und grammatische Form erkennen zu können) [...] Im Lichte der

${ }^{1}$ S. die Blending-Theorie von Mark Turner und Gilles Fauconnier (2002). 
anthropozentrischen Sprachtheorie beinhalten externalisierte Äußerungen weder ihre Bedeutungen noch die Sprache, auf deren Grundlage sie entstanden sind. Sprachliche Äußerungen sind lediglich substanzielle Exponenten eines bestimmten Wissensbereichs eines konkreten Menschen. (Bajerowska 2013: 26 f.)

Der Grundansatz der Emergenz besagt, dass zur Entstehung bestimmter Phänomene sog. „heterogen” wirkende Ursachen beitragen. Aus weniger komplexen Entitäten emergieren andere, die sich durch einen höheren Komplexitätsgrad auszeichnen. Nach Jürgen Mittelstraß (2005: 313) ist

Eine Wirkung [...] heterogen bzw. emergent, wenn sie nicht als Summe der an ihrem Zustandekommen beteiligten Kausalfaktoren verstanden werden kann. In gegenwärtiger Terminologie bezieht sich der Emergenzbegriff auf das Verhältnis der Eigenschaften von Ganzheiten zu Eigenschaften ihrer Bestandteile [...] Eine emergente Ganzheitseigenschaft unterscheidet sich qualitativ von den Eigenschaften der Komponenten und kann aufgrund prinzipieller Beschränkungen nicht angemessen auf diese Komponenteneigenschaften zurückgeführt werden.

Beim Aufspüren möglicher emergenter Eigenschaften der Textdenotate sind zwei subtile von John Searle (1999) herausgearbeitete Reduktionskategorien heranzuziehen: die ontologische und die kausale Reduktion. Die Emergenz innerhalb eines aus Teilen A, B und C bestehenden Systems manifestiere sich durch das Auftauchen neuer Systemeigenschaften, die keine der Systemkomponenten aufweist. Das Zustandekommen und die fortwährende Existenz systemischer Eigenschaften werde durch kausale Interaktionen zwischen den Systembestandteilen bedingt (vgl. Poczobut 2012: 96 f.). Ein Beispiel für kausal reduzierbare emergente Systemeigenschaften könnten meines Erachtens Textdenotate darstellen, deren Existenz und Eigenschaften kausale Interaktionen zwischen den einzelnen Neuronenkoalitionen zugrunde liegen. Nach Poczobut (2012) beruht das Wesen der kausalen Reduktion auf der Erklärbarkeit kausaler Kräfte der zu reduzierenden Entität mittels kausaler Interaktionen zwischen Entitäten, die die Reduktionsbasis darstellen. Ein Beispiel für die kausale Reduktion auf dem Gebiet der Linguistik stellt das Verhältnis der Textdenotate zu den relevanten neurobiologischen Vorgängen dar. Die letzteren liefern eine kausale Erklärung für die Eigenschaften, Existenz und die kausalen Kräfte der Textdenotate, z.B. für das Potenzial, bestimmte (Fach-)Wissenselemente zu verändern bzw. transformieren. Im Hinblick auf Searl's These zur Ontologie des Geistes (1999) sind Textdenotate kausal auf neuronale Prozesse reduzierbar. Das mentale Phänomen Textdenotat wäre dann ontologisch auf neuronale Prozesse reduzierbar, wenn Textdenotate neuronale Prozesse wären. Als kausal reduzierbare Eigenschaften menschlicher Gehirne können Textdenotate weder auf andersartige Eigenschaften zurückgeführt noch durch diese ersezt werden, z.B. durch Interaktionsmuster verschiedener Neuronenkoalitionen (s. Mittelstraß 2005: 313 ff.). Daraus ergibt sich, dass Textdenotate mittels neurobiologischer Termini nicht definierbar sind. $\mathrm{Zu}$ ihrer Entstehung kommt es meines Erachtens dadurch, dass auf einer niedrigeren (Beschreibungs-)Ebene des menschlichen Kognitionssystems, z.B. auf der Ebene neuronaler Interaktionen eine kritische Komplexitätsschwelle überschritten wird. 
Ich vertrete den Standpunkt, dass die mentalen Vorgänge, die der Textproduktion zugrunde liegen, aufgrund einer Sequenz „kritischer Phänomene” im Verlauf des jeweiligen Textformulierungsprozesses erfolgen. Die als "kritische Phänomene" bezeichneten Entitäten werden als eine Kumulierung diskreter, (Fach-) Wissensgenerierung bzw. -transferenz begleitener Veränderungen innerhalb der sprachlichen Prozesse im Gehirn eines Individuums aufgefasst.

Im Hinblick auf die Behauptungen von George Ellis (2006) wird hier angenommen, dass die Emergenz bestimmter Gehirnaktivitäten zu Textdenotaten auf nichtlinearen Wechselwirkungen zwischen ihnen selbst sowie zwischen ihnen und ihrer Umgebung beruht. Mit dem Ausdruck „Umgebung” werden Variablen bezeichnet, die sowohl interne als auch externe kontextuelle Einbettung des Auftauchens denotativer Textebene parametrisieren z.B. das (Fach-)Wissen des Textverfassers, sein emotionaler Zustand, seine Sprach- und Kommunikationserfahrungen usw. Der oben umrissene Ansatz stellt eine Anspielung auf das Konzept der kontextuellen Emergenz (s. Poczobut 2012) dar. Aus der hoch spezifischen kontextuellen Einbettung der Emergenz neuronaler Prozesse zu Textdenotaten ergibt sich nach meinem Ermessen die Spezifität der denotativen Textebene.

\section{Zum anthropozentrisch fundierten Spezifizität-Begriff}

Vor dem Hintergrund der anthropozentrisch geprägten theoretischen Annahmen bezüglich der denotativen Dimension eines jeden konkreten (Fach-)Textes wird im Folgenden eine gewisse Umformulierung bzw. eine modifizierte, ontologisch untermauerte Auffassung des Spezifizität-Begriffs vorgeschlagen. Im Spiegel des anthropozentrischen Ansatzes stellt die stark individuums- und wissensbezogene Ausrichtung in der bisherigen Herangehensweise einen zweifellosen Vorteil dar. K. von Heusinger (2012: 2) äußert sich zum Problem der Spezifizität wie folgt: „Spezifizität umfasst eine Reihe von Phänomenen, die sich vielleicht am Besten mit der ,referenziellen Intention' des Sprechers oder der Sprecherin und deren Wissen über den einzuführenden Diskursreferenten beschreiben lassen."

Weiter schreibt K. von Heusinger (2012: 14):

Ein zentraler Typ der Spezifizität (die „epistemische Spezifizität") lässt sich am Besten mit dem Konzept der Sprecherintention verstehen. In der spezifischen Lesart intendiert die Sprecherin, sich auf ein bestimmtes Objekt zu beziehen, während sie in der nicht-spezifischen Lesart keinen bestimmten Referenten meint, sondern die Eigenschaft hervorheben will, die durch die Nominalphrase ausgedrückt wird. Dieser Kontrast zeigt sich nicht (notwendig) in den Wahrheitswerten des Satzes, kann aber mit dem Sprecherwissen getestet werden. [...] Dieses Konzept von epistemischer Spezifizität lässt sich zu einer ,referenziell verankerten Spezifizität' $[\ldots]$ verallgemeinern.

K. von Heusingers Aussagen sind nach meinem Ermessen als ein klares Anzeichen dafür zu verstehen, dass aus der Fülle des durchaus individuell geprägten, 
hoch differenzierten kognitiven Vermögens der einzelnen Menschen ausschließlich die an Intentionalität bzw. Referenz gebundenen Phänomene in den Spezifizität-Begriff einbezogen werden können. Zweitens kommt im oben zitierten Aufsatz die mangelnde Unterscheidung zwischen den in Gehirnen konkreter Sprecher-Hörer internalisierten bzw. generierten Textdenotaten und der Äußerlichkeit (vgl. Warnke/Spitzmüller 2008: 7) d.h. der Äußerungsebene der auf ihrer Grundlage in materieller Form realisierten Texte zum Vorschein.

Im Gegensatz zum hier präsentierten Konzept K. von Heusingers erstreckt sich eine anthropozentrisch untermauerte Auffassung der Spezifizität in ihrem primären Sinne auf das gesamte Vermögen sprachlicher Eigenschaften eines Menschen. Damit kann festgehalten werden, dass Spezifizität einen Komplex personenspezifischer Gegebenheiten innerhalb des gesamten sprachlichen Vermögens eines konkreten Sprecher-Hörers darstellt. Bei genauerer Betrachtung lässt sich feststellen, dass sie spezifische, an die einzigartige Kommunikationserfahrung eines Individuums gebundene, kontextuell, situativ, illokutiv und pragmatisch geprägte, neurobiologisch determinierte Verlaufsmuster sprachgebundener Prozesse zwischen/ in den betroffenen Neuronen, Neuronengruppen bzw. Neuronenkoalitionen im Gehirn eines Menschen umfasst (s. Koch 2008). Ihre materielle, direkt beobachtbare Emanation stellt eine jede der Vertextungen, die jeweils durch den personenspezifischen Formulierungsstil sowie den Abweichungsgrad und -stil von der sog. Sprachnorm gekennzeichnet wird. In Anlehnung an die anthropozentrische Maxime, die neurobiologische Forschung (ebd.) sowie die oben bereits präsentierten Emergenz-Konzepte nehme ich an, dass das mentale Phänomen Spezifizität als eine sprachgebundene Entität im Gehirn eines jeden konkreten Menschen aus physikalisch begründeten Wechselwirkungen zwischen Neuronen emergiert. Obwohl die Spezifizität auf einem mit den Naturrechten völlig konsistenten Fundament basiert, ist sie durch diese Rechte nicht erklärbar.

Das Phänomen der Spezifizität geht unumstritten mit dem Bindungsproblem (s. Linz 2002) einher, dessen Kern die gemeinsame Verarbeitung der einzelnen Teilinformationen in relativ weit abgelegenen Teilen des Gehirns und ihre Integration (Bindung) zu einer kohärenten Wahrnehmung bildet (s. Linz 2002, Koch 2008). Bei Koch (2008) werden die Bindungsmechanismen dreifach eingeteilt. Der erste Bindungsmechanismus beruht darauf, die genetische Information sowie sinnliche Erfahrungen aus der frühen Kindheit zu speichern. Dies resultiert darin, dass bestimmte Neuronen für zwei oder mehrere Merkmale bestimmter Objekte direkt zuständig werden. Der zweite der erwähnten Mechanismen stützt sich auf schnelles, effizientes Lernen: unterliegen bestimmte Neuronen einem häufigeren Einfluss eines und desselben Reizes, kommt es zu einer Modifizierung auf der Ebene interneuronaler Verbindungen, was wiederum zur Entstehung einer direkten mentalen Repräsentation des betrachteten Objekts führt. Der dritte, spezifitätsrelevante Bindungsmechanismus ist weder lernbedingt noch epigenetisch determiniert. Er basiert auf einer hoch spezifischen, auf der Ebene einzelner Neuronen 
nicht vorkommenden Strategie, die darauf beruht, bei der Wahrnehmung erstmalig betrachteter Objekte bzw. beim Erkennen bisher nicht identifizierter Objektmerkmale, neue Neuronenensembles (auf funktionaler Ebene) dynamisch zu generieren. Derartige Neuronenensembles bilden keine dauerhaften Entitäten, der Bindungseffekt entsteht sehr schnell und erfordert konzentrierte Aufmerksamkeit (ebd.). Solche Reaktionspotentiale des Gehirns könnten nach meinem Ermessen die neurobiologische Grundlage für die Herausbildung neuer (Fach-)Textmuster und damit für die (Fach-) Wissenserzeugung darstellen.

Die Plastizität des Hirngewebes (s. Koch 2008: 196) sowie die Möglichkeit funktional kohärente Neuronenensembles dynamisch zu bilden, deutet auf die funktionale Elastizität des Hirngewebes hin. Das menschliche Gehirn verfügt über die Fähigkeit, sein auf struktureller Ebene relativ homogenes ${ }^{2}$ physikalisches Fundament (die Neuronen) zu einer hoch spezifischen, „raumübergreifenden” Reaktion auf neue Reize zu bringen. Diese neurobiologisch determinierte Eigenschaft des menschlichen Gehirns sehe ich als eine potenzielle Grundlage für die anthropozentrische Fundierung des Spezifität-Begriffs an.

Im Hinblick auf die Differenziertheit der Bedeutungszuweisungsprozesse sind Textdenotate als hoch spezifische Entitäten aufzufassen. In Bezug auf ihren neuronalen Hintergrund sind sie allerdings homogen aus der Sicht der Statistik. Statistische Homogenität bedingt die Regelmäßigkeit, eine gewisse Systematik menschlicher Kognition sowie verbaler Kommunikationsakte, sie determiniert die Sprachkonvention sowie die unerlässliche Algorithmik der Kognitions- und Kommunikationsprozesse. Daher scheint die Kategorie der statistischen Homogenität relativ stark mit dem Wissen über die Aussageform verbunden zu sein. Die Spezifität der Textdenotate wird wiederum als eine personenspezifisch verlaufende Funktion des Ablaufs emergenter Wissens- und Textgenerierungsprozesse über die Zeit aufgefasst. Der Geltungsbereich dieser Funktion, ihr Definitionsbereich bzw. die Domäne und ihr Wertebereich erstreckt sich von der Formdeterminante (des im Verlauf konkreter Textgenerierungsprozesse aktivierten Wissens über die Form) bis hin zur Bedeutungsdeterminante (des individuell geprägten semantischen Wissens des Individuums). Die Spezifität d.h. der jeweils personenspezifische, kontextbedingte Verlauf der erwähnten Funktion könnte als Indikator für die Qualität bzw. den Konsolidierungsgrad des (neu) generierten Wissens gelten. Um emergente Phänomene im Bereich sprachlicher Eigenschaften der Menschen zu erforschen, sollte man zunächst Differenzschwellen innerhalb des Funktionsverlaufs identifizieren. Anschließend wäre zu prüfen, ob sich irgenwelche Regelmäßigkeiten in Bezug auf Veränderungen im Bereich der Form-Bedeutung-Korrelation feststellen lassen. Es ist mit Nachdruck hervorzuheben, dass die Kategorie der Spezifität in das anthropozentrische Herangehen eingebettet ist. Eine beträchtliche Herausforderung im forschungspraktischen Sinn stellt hier die Übertragung der

\footnotetext{
${ }^{2}$ Im Sinne regelmäßiger Strukturiertheit des Gehirns in der Makroskala.
} 
oben dargelegten Annahmen auf die Korrelation zwischen der Ausdrucks- und Denotationsebene dar.

Zusammenfassend ist Folgendes festzuhalten: a) neuronale Prozesse, die den sprachlichen Eigenschaften der Menschen bzw. der Entstehung von Textdenotaten zugrunde liegen, sind homogen aus der Sicht der Statistik, d.h. ihr Ablauf bei verschiedenen Individuen weist keine beträchtlichen Unterschiede auf; b) auf der Basis statistisch homogener neuronaler Prozesse emergiert Spezifität als eine Eigenschaft, die den von einem jeden Menschen formulierten Texten einen individuellen Charakter verleiht. Vor dem Hintergrund der letzteren Feststellung scheint es angemessen, die Aussage Warnkes/ Spitzmüllers (2008: 7) zu zitieren: „Bedeutung ist damit immer spezifisch [...] und resultiert aus einer Kontextualisierung [...] im verstehensrelevanten Wissen, sie ist also auf das Prinzip der Spezifizität bezogen. Analysen der Diskurslinguistik nach Foucault konzentrieren sich schließlich auf die Emergenz von Bedeutung im Feld der konkreten Vertextungen."

\section{Zum anthropozentrisch begründeten Entropie-Konzept}

Ein weiterer, in die linguistische Forschung integrierter Begriff, der im Lichte des anthropozentrischen Ansatzes eine tiefgründig angelegte Re-Formulierung erfordert, ist die Entropie. Meines Erachtens wirft ihre anthropozentrisch fundierte Konzeptualisierung ein neues Licht auf das Problem der (Fach-)Wissensgenerierung.

Ausgehend von der Entstehung neuer Tierarten bis zum Auftauchen bestimmter Eigenschaften menschlicher Gehirne ist Emergenz als Effekt der adaptiven Selektion in Interaktion mit der Umgebung zu betrachten. Vor dem Hintergrund der oben erwähnten nicht linearen Emergenzprozesse ist das komplexe, nuancenreiche Zusammenspiel sprachlicher Eigenschaften eines Individuums sowie seiner Fähigkeit, Wissen zu produzieren bzw. zu transformieren, meines Erachtens als ein hochentropisches System anzusehen. Als Zustandsgröße der Thermodynamik bestimmt die Entropie, in welcher Richtung Prozesse ablaufen, die man an einem abgeschlossenen System ausführt. Sie stellt keinen wohldefinierten Begriff sondern eine nur schwer fassbare, nicht direkt messbare physikalische Größe dar. Als solche beschreibt sie das Maß für die Unordnung in einem abgeschlossenen System, genauer gesagt das $\mathrm{Ma}$ für die Unkenntnis des Zustands aller einzelnen Teilchen des Systems. In Bezug auf biologische Entitäten wird die Informationsentropie als „das Maß des Unwissens” bezeichnet (s. Jaroszyk 2014: 153 ff., Hames/Hooper 2012: 105). Daraus ist meines Erachtens abzuleiten, dass sich das Entropiekonzept auch auf den Bereich der Wissensgenerierung erstrecken kann. Den potenziellen Zielbereich stellt vor allem die Generierung des Fachwissens in Bezug auf bisher unerschlossene bzw. wenig erforschte Wirklichkeitsgebiete dar, deren Entitäten einer intensiven wissenschaftlichen Exploration unterworfen werden. Auf der 
Ebene der Kognition sowie der Bedeutungsgenerierung könnten nach meinem Ermessen bestimmte Mechanismen zur Verringerung der Entropie bzw. spezifische Mechanismen, die leere Räume füllen lassen, von besonderer Bedeutung sein. Die letzteren bestimmen meines Erachtens die jeweils spezifische, hauptsächlich auf intertextueller Ebene erfassbare Topologie der Textdenotate. Insgesamt führt dies zur Entstehung neuer Wissenselemente, die auf der Denotationsebene personenspezifisch konsolidiert werden, d.h. in das bereits existierende Ensemble von Wissensmechanismen im Gehirn eines Menschen eingebunden werden. Die direkte Anspielung auf die Topologie im exakten Sinne d.h. auf die gegenseitigen Transformationen geometrischer Objekte erwächst hier aus der Annahme, dass Textdenotate räumlicher Natur sein können, obwohl sie keine Substanz sind. Als solche könnten sie den Mechanismen topologischer Transformationen unterliegen. Aufgrund einer Textanalyse unter Berücksichtigung entropisch bedingter Exponenten auf intertextueller Ebene wäre es möglich, topologisch orientierte, linquistische Modelle sowie quantitativ begründete Theorien aufzustellen. Ein entsprechend ausgebautes und ,sensibilisiertes” System der dargestellten Mechanismen könnte eine Identifizierung und Verifizierung bzw. Falsifizierung von Informationskonstellationen ermöglichen, die durch einen hohen Unsicherheitsgrad gekennzeichnet sind. Die gegenseitigen Wechselwirkungen der erwähnten Mechanismen determinieren die Entstehung sowie die gegenseitige Positionierung der neurobiologisch fundierten Form- und Bedeutungsdeterminanten, d.h. der Faktoren, die auf die Bedeutungkonstitution und die Textform Einfluss nehmen.

Im Spiegel der anthropozentrischen Maxime ist festzustellen, dass der Entropie-Begriff in erster Linie auf die sprachlichen Eigenschaften eines konkreten Menschen bzw. Textdenotate, vor allem aber auf die in menschlichen Gehirnen internalisierten Mechanismen der Wissens-Dekonstruktion (s. Bajerowska 2014: 24 ff.) zu beziehen ist. In diesem Sinne ist anzunehmen, dass sich die (Fach-)Textkonstruierung im Kontext neuen (Fach-)Wissens auf Entropie-Mechanismen (zur Dekonstruktion des alten, nicht mehr aktuellen [Fach-]Wissens) stützt. Nach Entropie-Spuren ist meines Erachtens nicht im kontextabhängigen Informationsgehalt eines Wortes zu suchen, sondern in Regelmäßigkeiten innerhalb von syntaktischen, lexikalischen, stilistischen etc. Schwankungen auf intertextueller Ebene, die durch Veränderungen im Wissensstand (in Bezug auf einen konkreten Wirklichkeitsausschnitt) verursacht werden. Demnach wäre die Entropie als Maß für die Dekonstruktion bestimmter (Fach-)Wissenselemente während der (Fach-)Wissensgenerierung zu definieren.

\section{Fazit}

Zusammenfassend möchte ich das theoretische Problem, vor das Sambor Grucza (2008, 2013) die moderne Fachsprachenlinguistik gestellt hat, erneut ins Auge fassen. Im Spiegel des hier umrissenen Herangehens wäre anzunehmen, dass es 
infolge einer Aktivierung bestimmter fachsprachlicher Eigenschaften zum Emergieren bestimmter Fachwissenselemente kommt. Das Wesen der von Sambor Grucza dargelegten Zusammenhänge liegt meines Erachtens in der spezifischen Funktionsweise bestimmter sprachbedingter Mechanismen für Entropieabnahme. Diese wird determiniert durch das Zusammenspiel zwischen der Formdeterminante und der Bedeutungsdeterminante und zwar in Korrelation mit dem Präzisionsgrad bereits internalisierter Fachwissenselemente.

\section{Literatur}

Bajerowska, Anna (2013): Besonderheiten der aktuellen polnischen Fachsprachenforschung. In: Grucza, Franciszek / Spiegel, Heinz Rudi / Schneider-Mizony, Odile / Żmudzki, Jerzy (Hrsg.): Akten des XII. Internationalen Germanistenkongresses Warschau 2010: Vielheit und Einheit der Germanistik weltweit. Fachsprachen in Theorie und Praxis. Geschichte des Deutschen als Fremdsprachenunterricht weltweit/Geschichte von DaF weltweit. Theorie und Geschichte der Translationswissenschaft. Frankfurt am Main/Berlin/Bern/Bruxelles/New York/Oxford/Wien. S. $25-30$.

Bajerowska, Anna (2014): Transferencja wiedzy specjalistycznej. Warszawa.

Ellis, George Francis Rayner (2006): On the Nature of Emergent Reality. In: Clayton, Philip / Davies, Paul (Hrsg.): The Re-Emergence of Emergence. Oxford. S. 79-110.

Grucza, Franciszek (1983): Zagadnienia metalingwistyki. Lingwistyka - jej przedmiot, lingwistyka stosowana. Warszawa.

Grucza, Franciszek (1993): Język, ludzkie właściwości językowe, językowa zdolność ludzi. In: Piontek, Janusz / Wiercińska Alina (Hrsg.): Człowiek w perspektywie ujęć biokulturowych. Poznań. S. 151-174.

Grucza, Franciszek (1997): Języki ludzkie a wyrażenia językowe, wiedza a informacja, mózg a umyst ludzki. In: Grucza, Franciszek / Dakowska, Maria (Hrsg.): Podejścia kognitywne w lingwistyce, translatoryce i glottodydaktyce. Warszawa. S. 7-21.

Grucza, Sambor (2004): Od lingwistyki tekstu do lingwistyki tekstu specjalistycznego. Warszawa.

Grucza, Sambor (2008): Lingwistyka języków specjalistycznych. Warszawa.

Grucza, Sambor (2013): Kognitive Funktion von Fachsprachen als Untersuchungsgegenstand der Fachsprachenlinguistik. In: Gucza, Franciszek / Spiegel, Heinz Rudi / Schneider-Mizony, Odile / Żmudzki, Jerzy (Hrsg.): Akten des XII. Internationalen Germanistenkongresses Warschau 2010: Vielheit und Einheit der Germanistik weltweit. Fachsprachen in Theorie und Praxis. Geschichte des Deutschen als Fremdsprachenunterricht weltweit/ Geschichte von DaF weltweit. Theorie und Geschichte der Translationswissenschaft. Frankfurt am Main/Berlin/Bern/ Bruxelles/New York/Oxford/Wien. S. 31-36.

Hames, David / Hooper, Nigel (Hrsg.) (2012): Biochemia. Warszawa.

Heusinger, Klaus von (2012): Referentialität, Spezifizität und Diskursprominenz im Sprachvergleich am Beispiel von indefiniten Demonstrativpronomen. Vorversion. Stuttgart. https:/www. uni-stuttgart.de/linguistik/sfb732/files/heusinger-2012-referentialitaet-spezifizitaet.pdf.

Jaroszyk, Feliks (Hrsg.) (2014): Biofizyka. Warszawa.

Koch, Christof (2008): Neurobiologia na tropie świadomości. Warszawa.

Linz, Erika (2002): Indiskrete Semantik. Kognitive Linguistik und neurowissenschaftliche Theoriebildung. München.

Mittelstraß, Jürgen (Hrsg.) (2005): Enzyklopädie Philosophie und Wissenschaftstheorie. Bd. 2: C-F. Berlin/Heidelberg. S. 313-315. 
Poczobut, Robert (2012): Relacje psychofizyczne. In: Miłkowski, Marcin / Poczobut, Robert (Hrsg.): Przewodnik po filozofii umystu. Kraków. S. 85-124.

Searle, John (1999): Umyst na nowo odkryty. Warszawa.

Turner, Mark / Fauconnier, Gilles (2002): The Way We Think: Conceptual Blending and the Mind's Hidden Complexities. New York.

Warnke, Ingo H. / Spitzmüller, Jürgen (2008): Methoden und Methodologie der DiskurslinguistikGrundlagen und Verfahren einer Sprachwissenschaft jenseits textueller Grenzen. In: Warnke, Ingo H. / Spitzmüller, Jürgen (Hrsg.): Methoden der Diskurslinguistik. Sprachwissenschaftliche Zugänge zur transtextuellen Ebene. Berlin/New York. S. 3-54. 